\title{
Análisis de la tasa de detección de fetos macrosómicos mediante ecografía
}

\author{
María Jesús Teva G. ${ }^{1}$, Rosario Redondo A. ${ }^{1}$, Isabel Rodríguez G. ${ }^{1}$, Sara Martínez $C .^{1}$, \\ Mariam Abulhaj M. ${ }^{1}$ \\ ${ }^{1}$ Unidad de Gestión Clínica de Obstetricia y Ginecología. Hospital Universitario San Cecilio, Granada. España.
}

\begin{abstract}
RESUMEN
Antecedentes: La macrosomía fetal es clínicamente relevante debido a que se asocia a un incremento significativo de la morbilidad materno-fetal. La ecografía es el estándar dorado para la estimación del peso fetal y la valoración de su crecimiento. Tiene una sensibilidad de $21,6 \%$, por tanto la probabilidad de detectar macrosomía es baja para todas las fórmulas. El error entre el peso real y el estimado es 7,5-10\%, y en el caso de RN macrosomas éste puede llegar al 15\%. Objetivo: Analizar las tasas de detección de fetos macrosomas mediante biometría ultrasonográfica en la Unidad de Gestión Clínica de Obstetricia y Ginecología del Hospital Universitario San Cecilio, Granada, España. Método: Estudio retrospectivo de casos-controles. Se analizaron gestaciones únicas a término, comparándo peso fetal estimado ecográfico con el peso del recién nacido (RN). Se incluyeron 200 casos (criterio de inclusión: peso del RN $>4.000 \mathrm{~g}$ ) y 100 controles (criterio de inclusión: peso del RN 3.000-4000 g). Resultados: La incidencia de macrosomas fue del 7,5\%. El error medio en la estimación de peso fetal en los macrosomas fue de $577 \mathrm{~g}$. El error medio en el grupo control fue $206,6 \mathrm{~g}$, diferencia estadísticamente significativa $(p<0,05)$. Conclusión: Nuestros resultados muestran una tasa de error para la detección de macrosomía del 13,5\% (577 g), algo inferior a la de diferentes publicaciones. Debemos ser cautos al decidir la interrupción del embarazo por esta causa.
\end{abstract}

\section{PALABRAS CLAVE: Macrosomía, peso fetal, ultrasonografía, recién nacido}

\section{SUMMARY}

Background: Fetal macrosomia is clinically relevant due to its association with a significant increase in maternal-fetal morbidity. Ultrasonography is the gold-standard method to estimate fetal weight and growth assessment. It has a sensitivity of $21.6 \%$, therefore the probability of detecting macrosomia is low for all ultrasonographycs formulas. The error between the estimated and actual weight is $7.5-10 \%$, and in the case of macrosoma it can reach $15 \%$. Objective: To analyze the detection rate of macrosomia fetal by ultrasound examination at our Unit of Obstetrics and Gynecology, Hospital Universitario San Cecilio, Granada, Spain. Methods: This is a retrospective study of cases and controls. We analyzed singleton pregnancies comparing estimated fetal weight with the weight of the newborn. It included 200 cases (inclusion criteria: newborn weight $>4000 \mathrm{~g}$ ) and 100 control (inclusion criteria: newborn weight $3000-4000 \mathrm{~g}$ ). Results: The incidence of macrosomia was $7.5 \%$. The mean error in estimating fetal weight in the macrosomia group was $577 \mathrm{~g}$, while the mean error in the control group was $206.6 \mathrm{~g}$, the difference mean error was statistically significant ( $p$ $<0.05)$. Conclusion: Our results show an error rate for detection of macrosomia of $13.5 \%(577 \mathrm{~g})$, somewhat lower than those reported in different reviews. We must be cautious in deciding the termination of pregnancy by this cause.

\section{KEY WORDS: Macrosomia, fetal weight, ultrasound, newborn}




\section{INTRODUCCIÓN}

El Colegio Americano de Obstetras y Ginecólogos (ACOG) (1) define la macrosomía fetal como el peso al nacimiento igual o superior a 4.500 gramos, corregido según sexo y etnia. Afecta al $10 \%$ de los embarazos (2). Recientes estudios han evidenciado que la macrosomía se asocia a mortalidad fetal, miocardiopatía hipertrófica, trombosis vascular, hipoglucemia neonatal, desproporción cefalopélvi$\mathrm{ca}$, traumatismos durante el parto, hemorragia en el posparto y distocia de hombros (3). También es un factor predisponente de obesidad en la niñez y adolescencia (4).

Se han descrito como factores de riesgo de macrosomía: diabetes, peso materno, ganancia ponderal excesiva durante el embarazo, edad gestacional $(3-10 \%$ de los macrosomas nacen después de las 41 semanas), multiparidad, antecedente de feto macrosoma, sexo fetal. Sin embargo, la mayoría de los recién nacidos de $>4.500$ g no presentan ningún factor de riesgo (5).

El método para diagnosticar macrosomas consiste en calcular el peso fetal mediante ecobiometrías. La probabilidad de detectar macrosomía mediante dicha técnica en embarazos no complicados varía entre $15-79 \%$. Los métodos utilizados tienen una sensibilidad de $21,6 \%$, especificidad de $98,6 \%$ y valor predictivo positivo de $43,5 \%$ (6). El cálculo del peso fetal es menos preciso cuando se trata de fetos de gran tamaño con respecto a los de peso normal. Un peso estimado $>4.000 \mathrm{~g}$ se asocia con una probabilidad del $77 \%$ de macrosomía, siendo del $86 \%$ cuando sobrepasa los $4.500 \mathrm{~g} \mathrm{(7)}$.

Para todas las fórmulas ultrasonográficas consideradas, el error entre el peso real y el estimado es entre $7,5-10 \%$. El margen de error en caso de recién nacidos macrosomas puede llegar al 15\%. Hay un gran debate en la literatura respecto a la mejor fórmula ecográfica para predecir la macrosomía fetal. Las fórmulas ecográficas que se utilizan normalmente sufren grandes desviaciones cuando se trata de fetos macrosómicos $(8,9)$. Chauhan y cols (10), en 2005 realizaron una revisión de 20 artículos que calculan la sensibilidad y especificidad ecográfica para peso fetal estimado de $>4.000 \mathrm{~g}$ encontrando que puede variar ampliamente, desde $15 \%$ a $79 \%$. Hoopmann y cols (11), compararon la precisión de 36 fórmulas de estimación del peso de los fetos macrosómicos, llegando a la conclusión de que aunque algunas fórmulas presentaban ventajas, éstas no alcanzaban una tasa de detección de macrosomía fetal que llevara a una recomendación clínica (11). Melamed y cols (12), encontraron que los modelos basados en tres o cuatro índices biométricos parecían ser más precisos para el diagnóstico de macrosomía fetal que los modelos basados en sólo dos índices o que aquellos centrados en la circunferencia abdominal como única medida.
Varios autores han desarrollado fórmulas específicas para la estimación del peso del feto macrosómico teniendo en cuenta tanto la estimación de peso fetal mediante biometría como factores maternos informándose así mejores tasas de detección de macrosomía $(13,14)$.

El objetivo de este estudio fue analizar las tasas de detección de fetos macrosomas mediante la exploración ecográfica.

\section{MATERIAL Y MÉTODO}

Estudio retrospectivo de casos y controles, efectuados en recién nacidos de gestaciones únicas a término (entre la semana 37 y la 42) en el Hospital Universitario San Cecilio (HUSC) de Granada durante los años 2009 y 2010, ambos inclusive.

Criterios de exclusión: gestaciones gemelares, partos de pretérmino ( $<37$ semanas), partos $\geq 42$ semanas, muertes fetales anteparto y gestantes no controladas.

Grupo casos: 200 recién nacidos (RN) vivos macrosomas, definidos como $\mathrm{RN}$ con peso al nacer superior a $4.000 \mathrm{~g}$, y con peso fetal estimado (PFE) por ecografía en el tercer trimestre en nuestro Servicio. Consideramos pérdidas un total de 111 $\mathrm{RN}$ con peso mayor a $4000 \mathrm{~g}$ en los que no quedó constancia en la historia clínica de PFE por ecografía en el tercer trimestre.

Grupo controles: $100 \mathrm{RN}$ vivos durante el mismo período de tiempo cuyo peso al nacer entre 3000-4000 g, con peso fetal estimado por ecografía en el tercer trimestre. Este grupo se extrae del total de los recién nacidos entre 3000 y $4000 \mathrm{~g}$ en el mismo período, de forma aleatoria.

Los datos fueron obtenidos mediante la revisión de las historias clínicas recogidas en la base electrónica intrahospitalaria. El estudio fue aprobado por el Comité de Ética de la Institución.

Los pesos fetales son estimados mediante exploración ecográfica a partir de las medidas del diámetro biparietal, circunferencia abdominal y longitud del fémur. Se emplean para el cálculo del PFE las tablas de Hadlock predeterminadas en los ecógrafos de nuestra unidad, con un tiempo medio desde la realización de la ecografía y el parto de 7 días.

Se realiza un estudio descriptivo de nuestros datos analizando, tanto en el grupo de casos como en el de los controles, las siguientes variables: edad materna, fórmula obstétrica, antecedentes personales maternos y terminación de la gestación. Se realizó prueba de comparación de 2 medias para muestras independientes (Test de Student), considerándose como nivel de significación un valor $p<0,05$, con el objetivo de comparar el peso del RN con PFE en ecografía del tercer trimestre registrado en nuestra base de datos. Se analizan los datos mediante el paquete estadístico SPSS 15.0. 


\section{RESULTADOS}

Todos los resultados descritos fueron obtenidos de la base de datos electrónica intrahospitalaria del HUSC, que atiende a la población del área sur de Granada y es centro de referencia de otros hospitales de menor nivel asistencial.

En el período estudiado hubo un total de 5.576 partos. La incidencia de macrosomas fue de 7,5\% del total de recién nacidos. En el grupo de macrosomas encontramos que el $43,5 \%$ de los casos fueron embarazos cronológicamente prolongados (41-42 semanas) mientras que en el grupo control fueron el $24,7 \%$. La terminación del parto en el grupo de macrosomas fue espontáneo en el 51,5\% de los casos, cesárea en el 34,5\%, y partos instrumentales en el $14,5 \%$ de los casos. En el grupo control encontramos un total de $76,3 \%$ partos espontáneos, cesáreas en el $17,5 \%$ y $6,2 \%$ de partos instrumentales.

La media de edad materna fue 31,2 $\pm 4,9$ años y el $42 \%$ de las gestantes eran nulíparas en el grupo de casos, mientras que en el grupo control la media de edad materna encontrada fue 30,92 \pm 5,5 años siendo nulíparas el $43,3 \%$ de ellas. En ambos grupos la muestra se distribuye de manera homogénea en cuanto a edad materna y fórmula obstétrica.

Una de las variables analizadas fueron los antecedentes personales maternos más relevantes. En el grupo de macrosomas los más destacados fueron: diabetes gestacional $(7,5 \%)$, obesidad $(4,5 \%)$, diabetes pregestacional (3\%), antecedente de RN previo macrosoma (3\%), hipotiroidismo (3\%), hipertensión (2\%). En el grupo control evidenciamos $1 \%$ de diabetes gestacional, obesidad (1\%), sin encontrar ningún caso de diabetes pregestacional, y $7 \%$ de hipertensión. El peso medio del RN en el grupo de macrosomas fue $4.230 \pm 243,6 \mathrm{~g}$ (el caso de mayor peso fue $5.140 \mathrm{~g}$ ), mientras que en el grupo de controles el peso medio del recién nacido fue $3.411 \pm 267,5 \mathrm{~g}$.

Al realizar el test de comparación de dos medias para muestras independientes se revela que el error medio en la estimación de peso fetal en el tercer trimestre en el grupo de macrosomas fue de $577 \pm 324,8 \mathrm{~g}$, mientras el error medio en el grupo control fue $206,6 \mathrm{~g} \pm 372,9 \mathrm{~g}$, siendo la diferencia en el error medio estadísticamente significativa.

\section{DISCUSIÓN}

Nuestro estudio determinó una incidencia de $\mathrm{RN}$ con peso superior a los $4.000 \mathrm{~g}$ del $7,5 \%$, siendo la tasa de macrosomía a nivel mundial en torno al $10 \%(2,15,16)$. Las ecografías fueron realizadas por dos operadores diferentes que confirmaban la existencia de macrosomía fetal, realizándose dicha ecografía en un intervalo de una semana hasta el parto.
Así en nuestro estudio encontramos en el grupo de los macrosomas una tasa de cesáreas del $34,5 \%$ y de partos instrumentales del $14,5 \%$ que representan el doble de las encontradas en el grupo de controles. Asimismo supone un importante impacto en los servicios destinados a su atención, todo ello hace fundamental su detección antenatal.

La detección de los principales factores de riesgo de macrosomía fetal puede mejorar el diagnóstico y los resultados perinatales de estos fetos. El más importante de esos factores de riesgo es la patología del metabolismo de los hidratos de carbono de las embarazadas, ya sea como diabetes gestacional o pregestacional. En nuestro estudio hemos encontrado una tasa de diabetes gestacional del $7,5 \%$ y pregestacional del $3 \%$. Por tanto en la mayor parte de los RN con peso superior a $4000 \mathrm{~g}$ el cribado de diabetes gestacional mediante el test de O'Sullivan en el segundo trimestre fue normal. Esto debe llevarnos a replantear la estrategia para el diagnóstico de diabetes en el embarazo. Es muy importante concienciar de la necesidad de realizar el test de O'Sullivan en el primer trimestre a aquéllas gestantes con factores de riesgo para diabetes gestacional, tales como: edad mayor de 35 años, antecedente de macrosoma, obesidad, historia familiar de diabetes, pérdidas fetales.

Actualmente estamos reconsiderando los criterios diagnósticos de diabetes gestacional. Clásicamente si el test de O'Sullivan del I o II trimestre eran patológicos (resultado mayor o igual a 140) se realizaba la sobrecarga oral glucosa (SOG) con $100 \mathrm{mg}$, siendo los valores de normalidad: 105/190/165/145. Dos valores alterados se consideraban diagnósticos de diabetes gestacional. Nuevos estudios abogan por modificar estos criterios y así mejorar el diagnóstico precoz de la diabetes gestacional. De este modo también se definiría mejor el grupo de gestantes con riesgo elevado de presentar macrosomía fetal. En concreto nuestra Unidad de Gestión Clínica ha implantado junto con el Servicio de Endocrino del HUSC un nuevo protocolo para el diagnóstico y manejo de estas gestantes administrando $75 \mathrm{~g}$ de glucosa para la SOG y considerando como valores normales: 92/180/153, siguiendo las recomendaciones de la American Diabetes Association en 2011(15).

En nuestro estudio encontramos que hasta un $43,5 \%$ de los $\mathrm{RN}$ con más de $4.000 \mathrm{~g}$ presentaron un embarazo cronológicamente prolongado, lo que es en sí mismo un importante factor de riesgo de macrosomía. Por tanto es importante poder diagnosticar ecográficamente un feto macrosómico a la hora de tomar una correcta decisión obstétrica en relación con el momento y la vía de finalización de la gestación (3).

La ecografía es el método considerado estándar de oro para la estimación del peso fetal y la valoración del crecimiento. Tiene una sensibilidad de 
$21,6 \%(6)$, por tanto la probabilidad de detectar macrosomía es baja. Siendo el cálculo del peso menos preciso cuando se trata de fetos de gran tamaño con respecto a fetos de peso normal (7).

Se emplean distintas fórmulas para la estimación del peso fetal por ecografía, siendo las tablas de Hadlock las que han demostrado un menor margen de error $(9,20)$. Nuestros ecográfos tienen predeterminadas las tablas Hadlock para el cálculo del peso fetal a partir de la medida del diámetro biparietal, circunferencia abdominal y longitud el fémur. Por supuesto, es fundamental realizar estas medidas siguiendo los estándares de calidad establecidos (17). Un campo de mejora en este sentido puede ser entrenar las habilidades de los facultativos responsables de la ecografía del III trimestre de la gestación, así como que la estimación del peso fetal sea realizada por dos facultativos distintos antes de tomar una decisión en cuanto al manejo o el momento de finalización la gestación. Sin embargo, para todas las fórmulas consideradas, el error entre el peso real y el estimado no es inferior a 7,5-10\%, y en el caso de RN macrosomas éste puede llegar al $15 \%$. Nuestros resultados muestran una tasa de error para la detección de macrosomía del 13,5\% (577 g), algo inferior a la reflejada en diferentes revisiones publicadas a nivel mundial $(9,20)$.

Estamos obligados a realizar un análisis de las causas o antecedentes que pueden condicionar la macrosomía fetal y tomar las medidas que estén a nuestro alcance para reducirla, actuando sobre factores clínicos en los cuales podemos influir, como son: el embarazo prolongado, la diabetes, la obesidad, etc., estableciendo en cada caso su pronóstico para elegir la vía del parto más adecuada.

\section{CONCLUSIÓN}

La ecografía es el estándar dorado para la estimación del peso fetal y la valoración de su crecimiento. Nuestros resultados muestran una tasa de error promedio para la detección de fetos macrosomas del 13,5\% (577 g), por lo que debemos ser cautos en la toma de decisiones de terminar la gestación por esta causa (8).

\section{REFERENCIAS}

1. American College of Obstetrics and Gynecologist. Fetal Macrosomia. Practice Bulletin 2000; 22: 1-11.

2. Martin JA, Hamilton BE, Sutton PD, Ventura SJ, Menacker F, Kirmeyer S. Births: final data for 2004. Natl Vital Stat Rep 2006;55:1-101.

3. Mondestin MA, Ananth CV, Smulian JC, Vintzileos AM. Birth weight and fetal death in the United States: the effect of maternal diabetes during pregnancy. Am J Obstet Gynecol. 2002;187:922-6.
4. Carrera JM. Protocolos de Obstetricia Instituto Universitario Dexeus. 2003. Tercera reimpresión. Ed Masson, S.A.

5. Giusti SA, Yaccuzzi WJ, Balbuena LE, Torregrosa D, Elizalde MA. Complicaciones más frecuentes del recién nacido macrosómico. Rev Posgrado Vla Cátedra Med 2002;113:29-32.

6. Sadeh-Mestechkin D, Walfish A, Shachar R, Shoham-Vardi I, Vardi H, Hallak M. Suspected macrosomia? Better not tell. Arch Gynecol Obstet 2008;278:225-30.

7. ¿Qué es un feto macrosómico? Especialistas en diagnóstico ecográfico. 2010. Hallado en: http \:www.diagnus.com

8. Melamed N, Yogev Y, Meizner I, Mashiach R, Ben-Haroush A. Sonographic prediction of fetal macrosomia: the consequences of false diagnosis. J Ultrasound Med 2010;29:225-30.

9. Walsh JM, McAuliffe FM. Prediction and prevention of the macrosomic fetus. Eur J Obstet Gynecol Reprod Biol 2012;162:125-30.

10. Chauhan SP, Grobman WA, Gherman RA, Chauhan VB, Chang G, Magann EF, Hendrix NW. Suspicion and treatment of the macrosomic fetus: a review. Am J Obstet Gynecol 2005;193:332-46.

11. Hoopmann M, Abele $H$, Wagner $N$, Wallwiener D, Kagan KO. Performance of 36 different weight estimation formulae in fetuses with macrosomia. Fetal Diagn Ther 2010;27:204-13.

12. Melamed N, Yogev Y, Meizner I, Mashiach R, Pardo J, Ben-Haroush A. Prediction of fetal macrosomia: effect of sonographic fetal weight-estimation model and threshold used. Ultrasound Obstet Gynecol 2011;38:74-81.

13. Hart NC, Hilbert A, Meurer B, Schrauder M, Schmid M, Siemer J, Voigt M, Schild RL. Macrosomia: a new formula for optimized fetal weight estimation. Ultrasound Obstet Gynecol 2010;35:42-7.

14. Nahum GG, Stanislaw H. Ultrasound alone is inferior to combination methods for predicting fetal weight. Ultrasound Obstet Gynecol 2007;30:913-4.

15. Standards of Medical Care in Diabetes 2011. American Diabetes Association. Diabetes Care 2011;34:Suppl 1:S1-11.

16. Colman A, Maharaj D, Hutton J, Tuohy J. Reliability of ultrasound estimation of fetal weight in term singleton pregnancies. $\mathrm{N} Z \mathrm{Z}$ Med $\mathrm{J}$ 2006;119:U2146.

17. Fiestas C, Valera D, Palacios J, Gonzales L, Bardales B, Cisneros J. Comparación de dos fórmulas para calcular el peso fetal ecográfico vs peso al nacer. Ginecol Obstet 2003;49:214-8.

18. Albornoz J, Salinas H, Reyes A. Morbilidad fetal asociadas al parto en macrosomas: análisis de 3981 nacimientos. Rev Chil Obstet Ginecol 2005;70:218-24. 
19. Anderson NG, Jolley IJ, Wells J. Sonographic estimation of fetal weight: comparison of bias, precision and consistency using 12 different formulae. Ultrasound Obstet Gynecol. 2007;30:173-9.
20. Pinette MG, Pan Y, Pinette SG, Blackstone J, Garrett J, Cartin A. Estimation of fetal weight: mean value from multiple formulas. $J$ Ultrasound Med 1999;18:813-7. 